\title{
The Impact of Intelligent Medicine on Health Care against the Backdrop of Big Data
}

\author{
Min Shi , Zenghui Zhao \\ Xi'an Peihua University, Shaanxi Xi'an,china \\ 1243838277@qq.com
}

\begin{abstract}
Keywords: Intelligent Medicine(IM); Big Data; Artificial Intelligence(AI); Health Care; Information Technology (IT)
\end{abstract}

\begin{abstract}
The most prominent feature of the 21st century is the flourishing development of information technology(IT), the IT represented by artificial intelligence(AI) has had a profound influence on various industries. The health care will be the first to bear the brunt, suffered its impact is mainly reflected in the following aspects: Intelligent diagnosis in various fields of medicine will continue to be close to or exceed doctors; Surgical robots and other intelligent operating system on treatment, with its accurate, minimally invasive advantages will gradually replace some of the doctor's work; With the support of big data and cloud-computing for people's daily life health management, it will become a new trend in the future medical field. In addition, intelligent medicine(IM) will also accelerate the efficiency of drug discovery and drug development. On the basis of improving the level of personalized medicine and precision medicine, it can improve the level of personalized medicine greatly. Meanwhile, it will face social rules, habits and even ethics issues with the impact of AI on the medical industry.
\end{abstract}

\section{Introduction}

The vigorous development of information technology(IT) has brought about profound changes in society since the new century. New IT emerged based on the Internet of Things, big data, cloud-computing, and mobile Internet, affect people's daily lives profoundly. The closed integration of IT and medical care also promotes profound changes in the field of health care. Intelligent medicine(IM) has begun to play an increasingly important role in the field of medicine and health. $\mathrm{IM}$ is part of $\mathrm{AI}$, it use modern technology to simulate human perception for judgment, reasoning and processing. The emergence of IM makes doctors' operations easier, more accurate, and visible. As a result, the medical level has been greatly improved and patient satisfaction has been improved. It also changed people's understanding of health and opened up new prospects for human health and medical care. We will discusse the current status of IM in the field of health care in this paper to analyze the impact of IM on health care in the context of big data and further explore the potential problems faced in the development of intelligent health care.

\section{Application of ai in diagnosis and treatment}

\subsection{Intelligent Diagnosis: Fundamental "Technological Revolution"}

AI has entered the medical field and developed rapidly with the advancement of medical big data.Doctors use modern IT to collect and analyze a large amount of data and information, and then use AI machine learningand calculation methods to quickly identify the data basis for the case to 
make a highly accurate diagnosis and decision making. Intelligent diagnostic system can enhance doctors' diagnostic ability, improve diagnosis efficiency, and become an important auxiliary tool for medical diagnosis. At the same time, intelligent diagnosis has improved the quality of medical services in public health institutions and hospitals, promoted the development of public health and diseases management.In the near future, doctors also need to learn "ideals" from the machine, and even some medical specialties may be replaced by AI.In September 2016, an article in New England Journal of Medicine, which was the first Impact Factor in the medical community, pointed out that the field of health care will be divided into losers and winners in the field due to the involvement of machine learning, while pathologists and radiologists unfortunately become the former[1].

Intelligent diagnosis has been widely used in diagnostic fields such as cancer, neurological diseases, cardiovascular diseases, etc. At present, the most important thing is the diagnosis of cancer, which is also the key to the development of intelligent diagnosis.

At first, Intelligent diagnosis has a significant advantage in the diagnosis of cancer. As early as 2012, high school students in Wisconsin of the United States designed an algorithm to determine the location of breast cancer cells through AI and machine learning. The accuracy rate was as high as 96\%, which was much higher than that of specialists[2]. In 2017, Google Brain and Verily scientists developed AI products for breast cancer diagnosis, which can effectively determine the exact location of breast cancer cells. The accuracy is about $90 \%$, and the diagnostic accuracy of pathologists is only 70\%.AI goes beyond humans.In addition, the diagnostic accuracy of AI for lung cancer is as high as 90\%, while the diagnostic accuracy of doctors is only 50\%.A study in 2016 showed that the use of lung cancer genetic maps and database training computer software programscanidentify cancer-specific features that are difficult to detect with the naked eye, and have demonstrated that $\mathrm{AI}$ is more accurate than pathologists in evaluating lung cancer tissue sections[3].

Second, the intelligent diagnosis of nervous system diseases is booming. AI system through the deep learning of massive disease information, greatly enhance the efficiency of the diagnosis of neurological diseases, the diagnostic accuracy rate of $95 \%$ or more, equivalent to the level of a senior chief physician[4]. In 2017, the first "AI Research Center for Neurological Disease" in the world was established at Beijing Tian Tan Hospital, a national clinical research center for neurological diseases. It has the largest database of encephalopathy tissue samples and blood sample banks in the country and the world's leading deep learning technology. The team has developed head MRI and CT imaging AI diagnostic products. In the future, more AI applications will be presented, such as neurological pathological slicing artificial intelligence, intelligent care for patients with cerebral neuropathy, treatment plans based on patient medical data, and limb and speech rehabilitation training for neurological diseases.

Third, the intelligent diagnosis of cardiovascular diseaseis in the ascendant. As early as in 1997, an expert system for diagnosis of cardiovascular diseases based on waveform analysis has been implemented.The system is characterized by the fact that each disease is treated as a framework in the knowledge base, and multiple slots are placed on the framework to store all the symptom facts that may occur in the symptom. In each symptom fact structure, there is a credibility factor that reflects the credibility of this fact.

AI companies promote the rapid development of intelligent diagnostics, themost representative company is IBM. IBM Watson robot is in the field of intelligent diagnosis leading products. This system has been deeply cultivated in the medical field for many years. The Watson system mainly focuses on the field of cancer diagnosis. It also covers major diseases such as diabetes and heart disease, and its coverage is still expanding.It is worth mentioning that Watson's commercialization 
in China has begun to gradually land. Although it is still in the initial stage of application, it is worth looking forward to large-scale applications in the future.

\subsection{Intelligent Treatment: Da Vinci in Surgery}

On the basis of intelligent diagnosis, AI is also being applied in the therapeutic field, and the most notable of these is the surgical robot.Currently, surgical robots can assist doctors in performing surgery, especially in the field of minimally invasive surgery.Surgical robots are mainly divided into two categories: robots controlled by doctors and fully automated robots.In contrast, surgical robot systems controlled by doctors are more mature, mainly including Da Vinci robot, Verb minimally invasive surgical robot, MAKO robotic arm surgery auxiliary system.Da Vinci robots are the most widely used and have become one of the most advanced minimally invasive surgical systems in the world. Compared to Da Vinci robots, the advantages of Verb's minimally invasive surgical robots are reflected in price, volume, and functionality. At the same time, it abandons the practice of remote surgery and adopts close-fitting surgery, which makes it more convenient in operation.Many domestic and foreign medical institutions are constantly developing new types of surgical robot systems. However, current surgical robots still require doctors' control and manual operations, and no fully automated surgical robots have emerged, but they have shown great potential for development.

The Children's National Health System research team in the United States developed fully automated surgical robots to improve the efficiency and safety of surgery.In May 2016, the team announced the results of their research-STAR (Smart Tissue Autonomous Robot), which is an intelligent tissue autonomous robot[5]. This is the world's first autonomous robot that can handle soft tissue.At present, STAR autonomous surgical robot is gradually moving towards clinical practice. As a result, the "assistant status" of surgical robots will be fundamentally changed, and intelligent machines are expected to truly take over the work of surgeons and provide surgical treatment services to humans.

\section{Application of AI in health management}

Medical big data promotes the application of AI in the medical field. The current big data is mainly the information input of hospital diagnosis. The future medical big data will include people's daily health management. Through AI algorithms, people can accurately grasp the health status of individuals and respond to the development of infectious and seasonal diseases through big data.Relying on big data and algorithm technology, AI is closely integrated with human daily life to provide high-quality, intelligent and daily medical care services for humans.Judging from the current development, the development of AI in the field of health management mainly focuses on the following five aspects.

\subsection{Machine Learning and Nutrition Management}

In November 2015, an article published by Cell explained the positive significance of machine learning for nutrition[6]. The team conducted a standardized diet test on 800 volunteers and collected data on their diet, exercise, and sleep. The results showed that the same foods were consumed by different people and the responses were significantly different.Therefore, the dietary intake advice given through intuitive experience in the past is inaccurate.After learning by machine, they found that the machine learning algorithm gave more accurate nutritional advice and better control of postprandial blood glucose levels. Therefore, AI can help users perform accurate assisted analysis so that users can make more appropriate choices. 


\subsection{Big Data and Monitoring fulminant disease}

As early as 2008, Google had already launched a flu forecasting service. By detecting users' searches on Google, they could effectively track signs of influenza outbreaks[7].The Google Baseline Study hopes to build a huge human health database to find a completely healthy human genetic model. According to this database, as long asthe user's health data is found to be different from the model, Google will alert the user to possible health problems and prompt them to prevent them.Google Fit has developed a series of wearable devices that are constantly collecting massive amounts of biometric data and are combining with Google Baseline Study projects to provide more powerful applications[8]. It is not difficult to see that combining big data and internet technologies, we can conduct timely and accurate monitoring and prevention of certain infectious diseases, and after establishing some databases and intelligent analysis models, these activities will be more convenient and rapid.

\subsection{Electrocardiogram(ECG) automatic diagnosis technology and ECG real-time monitoring}

Cardiovascular and cerebrovascular diseases have become the disease with the highest incidence in China. ECG analysis helps early detection of heart disease and reduces the mortality of various heart diseases. ECG automatic diagnostic system monitors myocardial ischemia, arrhythmia, helps clinicians understand the changes in the patient's ECG in various situations (such as work, rest, activities, meals, sleep, etc.). It is of unique value to understand the chest pain, chest tightness, syncope, and other symptoms related to cardiac electrical activity. The ECG autodiagnostic device promotes the improvement of the family's health care and enables daily monitoring of heart health[9].

\subsection{Database Technology and Health Monitoring}

Germany's Nuritas Biotech combines AI with genetics to explore natural bio-organic factors that are conducive to health. In China, i Carbon X, an AI biotechnology company, is also trying to establish a healthy big data platform that uses its own and collaborators' power to collect people's various biological data. Ultimately, AI can be used to deal with this data to manage their health. Therefore, both the food database and the healthy big data platform is designed to monitor and record human health factors through big data and AI technologies, and to obtain more accurate and effective health management plans by analyzing these records and data.

\subsection{Health Management and Life Quality Improvement}

With the continuous improvement of people's living standards, the strict management of their own health will become the daily appeal of many people.If we can collect health data for every aspect of each person and use these data as a basis, the daily management of health can be easily achieved through AI algorithms. In recent years, the rapid development of Welltok is focused on improving personal health management and quality of life. The core philosophy of the company's services is that medical and health services are not only needed for patients, but also ordinary people need to pay attention to and maintain their own health. This shows that people's demand for their own health is higher, but also shows that the public's urgent needs for health programs, in addition to medical services also need to be complementary to the health services industry.

\section{Application of im in hospital management}

\subsection{Relieve the conflict between doctors and patients}

IM has effectively increased medical resources and alleviated the contradiction between doctors and patients, especially in countries where China's medical resources are unevenly distributed. Contradictions between doctors and patients are not necessarily due to incorrect diagnosis or treatment failure. The main reason is that the services provided by the hospital cannot meet the 
requirements of the patients, or that the patients cannot get the medical resources they deserve or ideal. From the hospital's point of view, the contradiction between doctors and patients often arises because medical resources cannot be used efficiently and reasonably, thus reducing the level of service they deserve. If in the course of diagnosis and treatment, it is possible to make full use of the ancillary services and operational arrangements of AI, not only can the strength of medical services be supplemented, but also medical resources can be more reasonably allocated and the efficiency of treatment can be improved. For example, using big data analysis, AI can arrange the time for treatment and treatment with reasonable and peak dislocation. This can not only reduce the time for patients to queue up, but also improve the efficiency of appointments and visits, and reduce the pressure on medical staff to allow them more time to treat patients. At the same time, AI is also used in many other aspects in hospitals, making procedures such as registering, asking for directions, paying bills, and printing assay reports more convenient and quick. The improvement of the efficiency of treatment can greatly improve the patient's satisfaction, which effectively reduces the occurrence of contradictions between doctors and patients.

\subsection{Improve the utilization efficiency of medical resources}

At present, AI products have entered the hospital management and services. In terms of future development trends, the AI application scenarios related to hospital management will mainly focus on two major areas. One is the virtual medical assistant and the other is the intelligent decision making of the hospital. Virtual medical assistants can provide supplementary medical advice, health care, and case tracking services in addition to doctors' treatment. This is equivalent to a "virtual nurse". It can play an important role in shunting the patients in the hospital, and the patients do not have to go to the hospital for treatment. The hospital's intelligent decision-making aims to build the hospital's decision-making process on the basis ofAI, so as to better improve the utilization efficiency of medical resources and the degree of intelligenceof hospital operations.

\subsection{Achieve IM decision-making}

Another important application of AI is hospital intelligent decision-making, eg. Analytics MD company of U.S. can provide hospital intelligence decision analysis system technology, its team has a wealth of medical health and big data processing experience. The Saasoftwaredeveloped by the company can collect detailed medical data from the U.S. government medical website and conduct an intelligent analysis of the data to provide doctors with the most reasonable advice, so that they do not need to repeatedly study complex casesto timely provide patients with the most appropriate treatment and services. This service can improve the quality and efficiency of patient and medical staff visits, and the availability of free medical resources can be rapidly utilized to avoid unavailability of resources and the asymmetry between doctor-patient services and demand. Therefore, hospital intelligent decision-making can exert many positive effects, which can not only improve the efficiency and satisfaction of patients' medical treatment, but also reduce the consumption of medical resources and control the medical costs[10].

The impact of AI on health care, in addition to the above-mentioned intelligent diagnosis, intelligent treatment, health management and medical management, will also play a significant role in such areas as drug discovery, biotechnology and precision medicine. In the screening of new drugs, the most secure compounds were selected as the best alternative to new drugs from tens of thousands of candidate compounds using the strategic network of the AI, the evaluation network, and the Monte Carlo Tree Search algorithm[11]. In cancer diagnosis and treatment, big data and AI can detect different lesions in different cancer patients or detect different lesions in different populations, formulate personalized medication plans, and improve the level of personalized medicine. Drug-taking and dispensing programs based on big data platforms will also greatly 
reduce the cost of drug use and increase efficiency. According to this trend of development, humankind's dream of subduing and even overcoming cancer in the future will eventually come true. In short, AI will more effectively promote the medical field to the direction of intelligence, daily life and humanity.These changes will have an important impact on public health and human understanding of itself.

\section{Potential problems of im}

Just as a coin has two sides, the emergence of new things will inevitably bring about some new problems.

First, people will face social rules, habits and even ethical issues. Big data is bound to involve the privacy of patients, and how to coordinate the tension between privacy protection and data acquisition is an important issue for IM. Social issues and regulatory issues need to be addressed. To gain people's trust, it is necessary to effectively supervise the use of AI health data and algorithms, and formulate relevant laws and regulations. Humans are expected to use artificial intelligence to overcome the cancer problem. The key technologies are focused on gene editing. Some large biotech companies hope to use this technology to slow down or stop human aging. The realization of this goal not only has to face many technical problems, but also has to face the insurmountable human ethical issues. If human beings acquire a certain degree of "eternal life", then the ethic and rules of human society will undergo revolutionary changes.

Second, the existing education and employment in the medical industry will be greatly affected.While improving the health and quality of life, IM will also have a certain impact on the medical industry. Many of the jobs previously performed by doctors, nurses, and managers may be replaced by AI. When the diagnostic accuracy of AI reaches or even exceeds that of doctors, people will gradually choose AI. Surgical robots can perform difficult procedures that are difficult for doctors. The application of intelligent hospital management can replace manpower and optimize patient treatmentprocesses, thereby effectively reducing the number of hospital management staff. Therefore, the above-mentioned three areas of practitioners will face enormous competitive pressure, and the corresponding medical education model will also need to adapt to the development of the new situation in order to respond to the popularization and application of artificial intelligence.

Finally, new technologies can only be widely used to reduce costs. In the context of the rise of artificial intelligence, roboticsurgeryhasa history of approximately 30 years of R\&D and application. However, the high cost of surgery has made people daunting. The huge $R \& D$ cost has seriously affected the widespread use of IM. With the rise of a new round of AI technology, surgical robots are also facing new developments. It can be expected that its cost will gradually decrease with the popularity of technology so that more people can benefit. At the end of 2017, Nanjing launched the R\&D project of "Hua TuoDr.Hwa", an intelligent doctor named after the ancient famous doctor Hua Tuo. This product is expected to provide efficient and inexpensive IM self-service for multi-levelusers, assisting in diagnosis and treatment.

\section{Summary}

The development of IM makes AI become a perfect medical expert at an amazing speed, and thus has the possibility to replace doctor in the future. As the robot has many advantages over the human brain such as precision and minimal invasiveness, it will lead to leapfrog development and transformation of the medical and health field operation model, and bring more patients and doctors 
a gospel of diagnosis and treatment. The important mission of AI is to improve medical efficiency and explore "incremental" in the existing medical resourceenvironment. By rapidly replicating the knowledge and diagnostic experience of top medical experts, we will train more "AI medical experts" that simulate the path of specialists toimprove the health care level. The in-depth application of AI in the medical field has become overwhelming. In this process, AI can effectively improve the overall medical level and health status of human beings, but it also brings certain social challenges and impacts. This is, to a large extent, a proof of the assertion that "technology is a double-edged sword". However, people can properly cope with the progress brought about by AI by properly planning and coordinating, and correctly respond to the impact of smart medicine on human society.

\section{Reference}

[1]Obermeyer Z, Emanuel EJ. Predicting the Future-Big Data, Machine Learning, and Clinical Medicine[J]. The New England Journal of Medicine, 2016, 375(13): 1216-1219.

[2]Wu Jun. Intelligent era[M]. Beijing: CITIC Press Group， 2016.

[3]Yu KH, Zhang C, Berry GJ, et al. Predicting non-small cell lung cancer prognosis by fully automated microscopic pathology image features[J]. Nature Communications, 2016, 7: 12474.

[4]Esteva A, Kuprel B, Novoa RA, et al. Corrigendum: Dermatologist-level classification of skin cancer with deep neural networks[J]. Nature, 2017, 542(7639): 115-118.

[5]Shademan A, Decker RS, Opfermann JD, et al. Supervised autonomous robotic soft tissue surgery[J]. Science Translational Medicine, 2016, 8(337): 337ra64.

[6]Zeevi D, Korem T, Zmora N, et al. Personalized nutrition by prediction of glycemic responses[J]. Cell, 2015,163(5): 1079-1094.

[7]Dugas A F, Jalalpour M, Gel Y, et al. Influenza forecasting with Google Flu Trends[J]. Plos One, 2013, 8(2): 1-7.

[8]Swan M. The Quantified Self: Fundamental disruption in Big Data Science and Biological Discovery[J]. Big Data, 2013, 1(2): 85-99.

[9]Song Yuning. Research and implementation of arrhythmia intelligent diagnostic System[D]. Northeastern University, 2010.

[10]Vockley M. Game-changing technologies: 10 promising innovations for healthcare[J]. Biomedical Instrumentation \& Technology, 2017, 51(2): 96-108.

[11]He Xianzhong, Xu Zhongxing, Chen Xin. Discussion on intelligent medical treatment caused by Human-Computer War [J]. Chinese Medical Engineering, 2016(4): 35-37. 